\title{
Measurements of Corona Discharge in Sharp -to-Plane Electrodes Configuration
}

\author{
Hassan J. Mohammed, Habeeb S. Jasim \\ Department of Materials Engineering, Engineering College, University of Diyala \\ Hassanjasim08@gmail.com, habeeb-shala@yahoo.com
}

\begin{abstract}
A Dc positive sharp- to -plane Corona phenomena have been studied in electronegative gases (air , and $\mathrm{SF}_{6}$ ) for pressure up to (3.2bar and gap length from 0.1 to $0.5 \mathrm{~cm}$ ), with high degree of non-uniformity electric field. The corona -breakdown phases can exhibit anomalous characteristics. The exact physical process of such phenomena is not yet fully understood complete. To better understand this process precise knowledge about corona and spark discharge is necessary. Hence the dependency of corona inception and spark breakdown voltage on the pressure, nonuniformity field factor and the type of applied voltage ,also the type of materials.

The steady region of corona discharge is observed in the ( sharp- to- plane ), for applied voltage between the threshold value voltage and onset value, we observe an effect that attaching gases. A Townsend type of discharge develops in the region close to point. At voltages above the onset value, the burst pulses are replaced by a Dc current, and in some cases 'pre-breakdown ' streamer are also observed.
\end{abstract}

Keywords: Stabilization corona discharges ,Sharp-to-Plane, Sulfur Hexafluoride.

Paper History: Received: (16/3/2017), Accepted: (16/5/2017)

\section{Introduction}

In a uniform electric field spark gap, when the technical conditions have been fulfilled for breakdown a highly ionized conducting channel propagates rapidly across the gap until it bridges the electrodes, and breakdown ensure. In non-uniform field gap, the conditions for breakdown may be fulfilled within a region where the applied electric field strength is high, but the ionized channel may not be able to propagate through the region where the field is lower. In such cases a corona discharge (or plasma)-occurs [1, 2, 3]. In our discussion of corona mechanisms, below ,we use the term streamer to describe aluminous conducting channel that is believed to propagate by photoionization and space charge distortion of the applied field.

In first stage of the study, a great deal of the efforts were stressed on the characteristics of very non uniform field gaps, whose breakdown voltage has unusual negative slope upon gas pressure $[4,5,6]$. Breakdown field strength of uniform field gaps in SF6 were, of course, another point of interest as a basic insulation property of the gas [5]. Research efforts were extended to more general factors which influence the breakdown characteristics of the gas , such as the shape of the electrode, electrode materials impurities ,applied voltage wave form, breakdown time lag etc [7]. A number of data of large scale experiments up to the extra -high voltage level are now available in public. In spite of all these research efforts, the understanding of the breakdown characteristics of SF6 is still far from complete. Although the attempts to formulate breakdown voltages of simple gaps as a function of the geometrical configuration.

One of the fundamental design problems of high -voltages transmission lines and switching stations is to determine the breakdown strength of a long non uniform field gap under a switching surge voltage. The physical processes leading to the breakdown of long air gaps and the theoretical mathematical modeling of these processes is extremely difficult $[7,9]$.

The main reason behind the statistical behavior of the corona -inception values is be the random manner in which a triggering electron could exist in the gap. For the corona inception to occur, the triggering electron must exist at a position where the electric field is high enough to produce effective ionization and lead to the formation of an avalanche of critical size. Once the avalanche of the critical size is formed, the excitation of the gas molecules in the primary avalanches. All of these secondary avalanches combined together will also be of the critical size. This is considered to be the criterion for corona inception. 
In the experimental work to determine the corona inception voltage and current pulses, the position of triggering electron is not known. The triggering electron could exist anywhere between a minimum distance and maximum distance from the anode. For the different positions of the triggering electron , there will be different magnitude of the corona -onset potentials.

In present paper the minimum magnitude of the corona onset potentials and current pulses are determine. The sharp -to plane geometry is considered or used and an Dc voltages of positive polarity is applied to the sharp electrode .From sharp electrode. This is the distance required for the triggering electron to produce a critical avalanche and hence the corona inception. Hence, the phenomenon of corona onset may be satisfactorily investigation using an electrode with relatively small radius of curvature $[8,10]$.

\section{Experimental detail}

The results presents below were obtained using a sharp-to-plane gap with a stainless steel materials, the sharp electrode was a $(0.05 \mathrm{~mm})$ a tip approximate of a radius space and from (1 to $5 \mathrm{~mm}$ ) away from a stainless -steel plane electrode, that the diameter is $(8 \mathrm{~cm})$ and $10 \mathrm{~mm}$ thickness. The apparatus employ for the present experiments is identical with described in the same as that of [11], but difference in the gap geometry. The corona phenomena were recorded for positive sharp DC conditions in SF6 gas by using ( High voltage generator up to $110 \mathrm{KV}$ ).

The experimental work have been carried out at atmospheric and ambient temperature using also the humidity, the instruments of experiments shows in diagram of Figure 1, and operational gas pressure between (1 bar and 3 bar). These pressure values allow to operate the chamber with a sparking parameter $(\mathrm{Pd})$ ranging from (1bar.mm to 15bar.mm).The reactor consists of a system of stainless steel sharp-to plane electrodes and the plasma source consists of a discharge chamber made of insulating materials, such as ceramic and Teflon. The pressure in the chamber was controlled by manometer connected to reactor from terminal and to the earth from anther terminal with resistor $(50 \Omega)$ for measurement.

The dependence of the' burst' pulses current discharges $I / d$ (where $I$ is the Trichel pulses current and is the length of gap between two electrodes collecting current ) on the voltage $\mathrm{V}$ applied on electrodes was measured .Both the breakdown with corona onset voltages and current pulses have been acquired a digital oscilloscope detectors. The DC high voltage was applied to sharp terminal and the dynamic processes in the ionization region and burst pulses measuring by multi-meters.

During all the experiments reported here, the electrodes have been cleaning and polishing for all series of tests that performed. The measurements carried out in "Diyala Electricity Distribution Directorate", for their assistance and support.

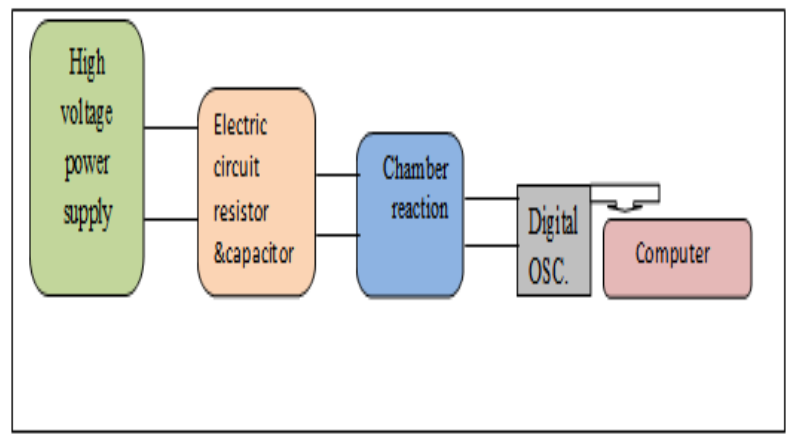

Figure 1: Simple schematic diagram illustration experimental apparatus used in this work

\section{Results and Discussion}

Many experimental data have been reported on the breakdown characteristics of $\mathrm{SF}_{6}$ some are relatively large scale experiments aiming the direct application to the design of high voltage gas insulated apparatus. Some experiments are performed on small scale gaps using relatively low applied voltage [12]. Even with the refined test conditions of these Laboratory experiments, the results are not consistent to each other particularly at high gas pressures. The low -field region is of great importance in the mechanisms of corona discharges :although it contribute to discharge growth directly, it acts as a storage space for ions (positive or negative ) whose space charge fields modify the events that occur in the high -field region. We observed from experiments, in nonuniformity geometry that in attaching gases corona inception potentials may be start separate from complete breakdown voltages . In the present work the non-uniformity factor in low pressure $\mathrm{SF}_{6}$, a discharge started at the point in the highest $(\mathrm{E} / \mathrm{pd})$ does not develop the breakdown of the gap. The discharge produces steady corona. Breakdown voltage of the gap is quite high in this case due to the deconcentration of electric field by the corona discharge, the phenomena is called as "corona stabilization"[11].

The high attachment property of $\mathrm{SF}_{6}$, due to the strong electron affinity, and electron attachments that occur in $\mathrm{SF}_{6}$ ( $\mathrm{SF}_{6}$, other dynamic processes that products such as SF5 and $\mathrm{F}^{-}$, and cluster ions ) by inelastic processes is very important. 
The results determine the $\mathrm{V} / \mathrm{Pd}, \mathrm{V} / \mathrm{d}$, and current -voltage characteristics in gap geometry, any electron in the gap will go to the point along field line and produce avalanche by collisional ionization, the space charge field due to the applied field is opposite therefor the primary avalanches will be stopped .The ions will become a part of the external circuit.

A present paper describes the experimental details and the results of measurements on the [ V-P,V-d, and I-V].

For attaching gases $\left(\mathrm{SF}_{6}\right)$, the negative ions go to the (+ tip) along the field lines and they are slower (1/100 ratio of velocity compared with the electrons), and the secondary electrons are formed by photoionization rather than by cathode processes.

Figure 1 illustrates the block diagram for the experimental instruments that used in the present work, the behavior that corona precedes breakdown at gas pressures (p) below some critical value $\left(\mathrm{p}_{\mathrm{c}}\right)$, and for higher pressures the curve of spark over voltage $\left(\mathrm{V}_{\mathrm{S}}\right)$ against $(\mathrm{p})$ is a continuation of the curve of corona threshold voltage against (p).

Figure 2 appeared relations ship between the breakdown and corona onset potentials versus of (pressures) with gap length of $(5 \mathrm{~mm})$ shows that, while the range of the type corona increasing linearly with voltage increases also breakdown occur, we observed that there difference between corona onset and breakdown voltage , and $\left(\mathrm{P}_{\mathrm{c})}\right.$ is not appear , because of electrodes configuration, that meant by curvature and area of electrode. The steady pre-breakdown corona discharge region observed in the (tip to plane ), and these are illustrated in Figures 3, 4, 5, 6, 7 \& 8. In the same range of applied voltages, we sometimes observe also 'pre-onset streamer ' which manifest themselves as luminous filaments propagating outwards from point. These streamers do not lead to breakdown because the field strength in the low-field region of the gap is too low to support their propagation. The glow corona is responsible for the stabilization process, and breakdown in the high part of the voltage/pressure curve is determine by a minimum field needed for streamer propagation.

The results suggested that, in the high and low part of the V/P curve, breakdown takes place when $(\mathrm{V} / \mathrm{pd})_{\min }$ and maximum ,this is necessary for streamer propagation. Since the value of $\mathrm{E} / \mathrm{P}$ (electric field /pressue) is largely controlled by corona space charge, differences in the sharp conditions do not affect the breakdown voltage in this curves. This explains why the breakdown voltage is to variation in the curvature of tip electrode, despite the large changes in tip field which result is very different corona onset values, that difference from results of [13], use rod to plane gap.

It also explains why, in gas having the unsimillarly curvature of electrode and materials made it ,as will as the gap length (d). This appeared very well in a Patchen's curve ,the breakdown voltage increases with d despite the small difference on corona onset ,such as shown in Figures 3, 4, 5, 6 \&7, from Figures, we illustrate that the corona onset voltage disappeared in low pressure and voltage (atmosphere air ), and appeared damage in curve, due to the electron attachment and ionization dynamic processes.

The measurements of current impulses can become assigned to lighting appearances in each case. Under certain experimental conditions of pressure and electrode length , the results demonstrate a conspicuous deviation from Patchen's law due to dominating streamer mechanism and electron attachment processes .On the other hand, The results of the corona pulses that mean the corona current then consists of pulses, known as Trichel pulses, as shown in Figures $8 \& 9$, these pulses typically have a short rise time and a relatively slow decay and they occur at a regular repetition rate for a fixed applied voltage, with increasing voltage, their repetition rate increasing , and when the onset voltage $V_{0}$ is reached they give way to a dc discharge. For all pressures below $\mathrm{Pc}$, the corona current at onset is in the form of individual avalanche pulses. These give ways at higher voltages to 'bursts ' of current of amplitude $250 \mu \mathrm{A}$ lasting typically a few milliseconds and, at a transition voltage shown by curve shown in figures, the large current pulses occurs at the beginning of each current burst. The current -pulse records has shown that the "burst" in the Figure 11.

The records show that each current burst is associated with a single one of the many discharges shown in the still photographs of the type corona, Figures $8 \& 9$ represented the comparison of current -voltage characteristics for different pressure or (distance), for positive corona discharge was appeared the current increases slightly with potentials discharges. Its appear high corona currents comparison with other experimental data [11]. The difference between them ,that the tip sharp is given large current and low corona onset and breakdown voltages compare with other results, because of the area of electrodes, when the area is small there are a high current.

Figure 10, illustrated the inception and breakdown potentials for non-uniform field geometry, and shows streak records and associated current pulses for inception corona in $\mathrm{a} \mathrm{SF}_{6}$ gas with the streak initial at beginning of the burst the photograph show that each current pulses. 


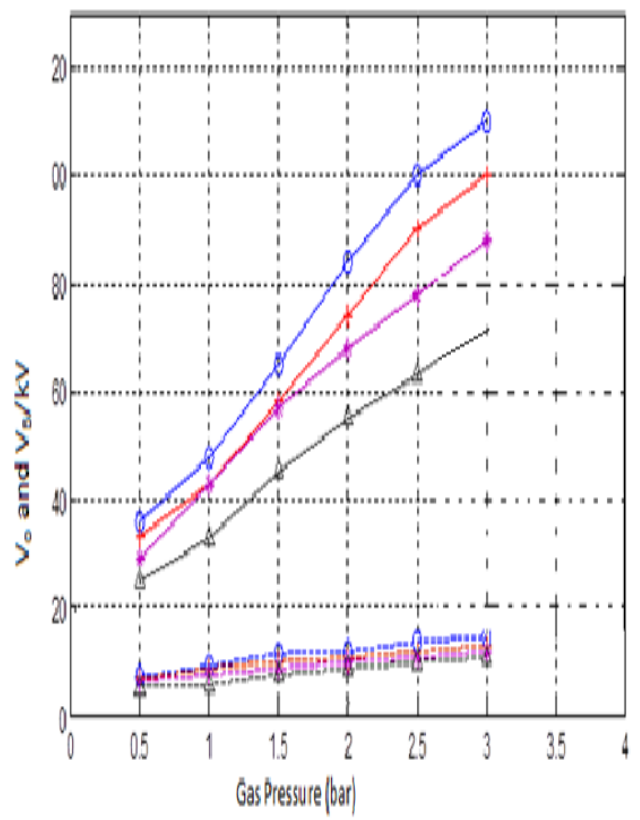

Figure 2: Positive sharp -to - plane corona characteristics with transition to breakdown inSF6 at ( O ) $\mathrm{d}=5 \mathrm{~mm},(+) \mathrm{d}=4 \mathrm{~mm},(*)$ $\mathrm{d}=3 \mathrm{~mm},(\Delta) \mathrm{d}=2 \mathrm{~mm}$

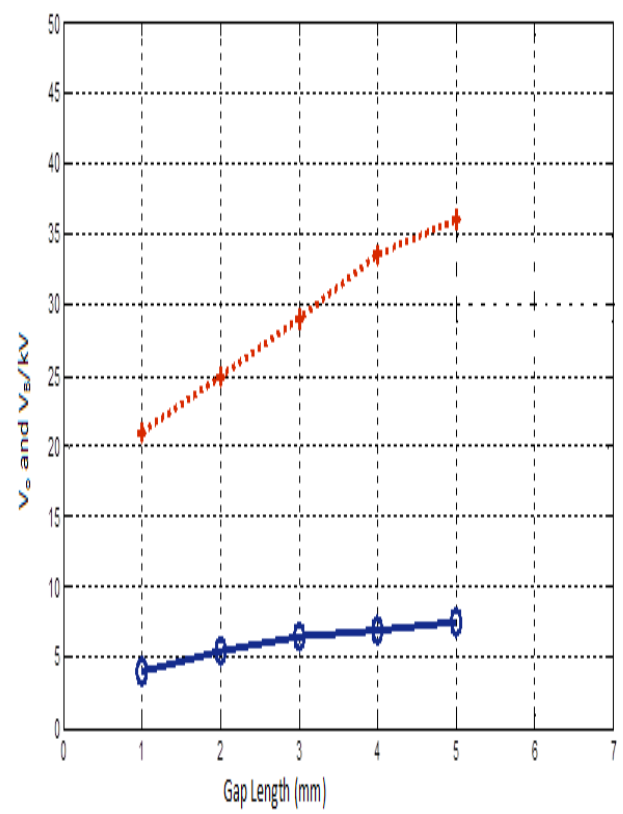

Figure 3: Electrical breakdown voltage $(+)$ $\&$ inception corona $(\mathrm{O})$ versus Gap length in SF6 gas with pressure 0.5 bar and atmosphere

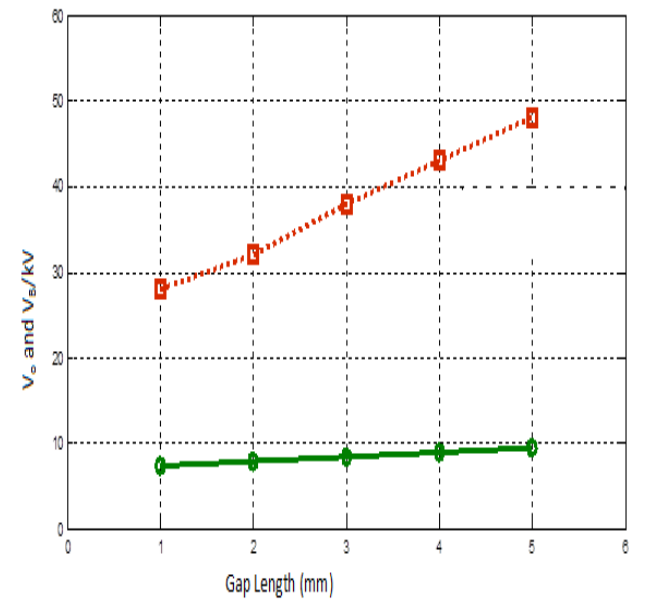

Figure 4: Electrical breakdown voltage ( $\square)$ \& inception corona $(\mathrm{O})$ versus Gap length in SF6 gas with pressure 1 bar and atmosphere

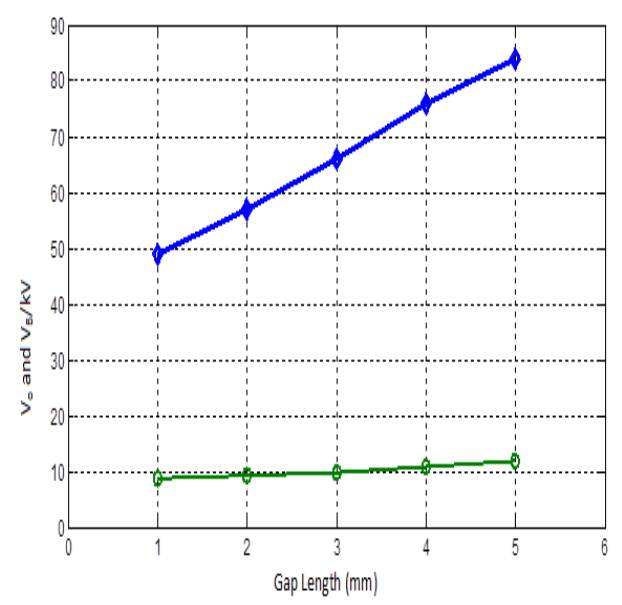

Figure 5: Variation in corona inception voltage $\mathrm{V}_{\mathrm{o}}(\mathrm{O})$, and spark potentials $\mathrm{V}_{\mathrm{B}}(\diamond)$ in SF6 with pressure 2 bar and atmospheric

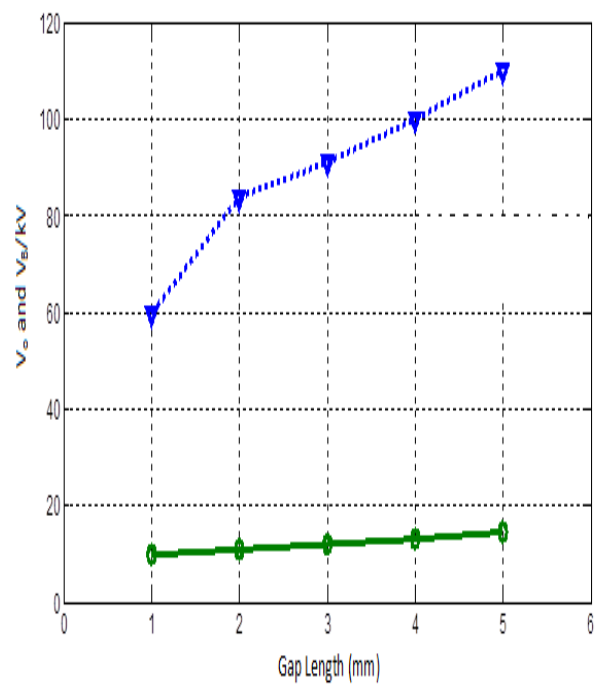

Figure 6: Variation in corona inception voltage $\mathrm{V}_{\mathrm{o}}(\mathrm{O})$, and spark potentials $\mathrm{V}_{\mathrm{B}}(\boldsymbol{\nabla})$ in SF6 with pressure 3bar and atmospheric 


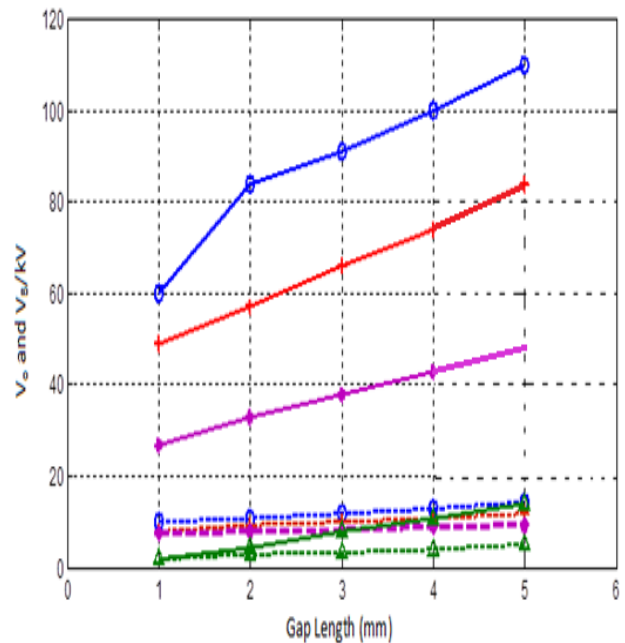

Figure 7: Variation in corona inception voltage $\mathrm{V}_{\mathrm{o}}$, and spark potentials $\mathrm{V}_{\mathrm{B}}$ in $\mathrm{SF} 6$ with pressure $(1,2,3$ bar $)[(\mathrm{O})$

$\mathrm{p}=1 \mathrm{bar},(+)=2 \mathrm{bar},(*) \mathrm{p}=1 \mathrm{bar},(\Delta)$ in air], and atmospheric pressure

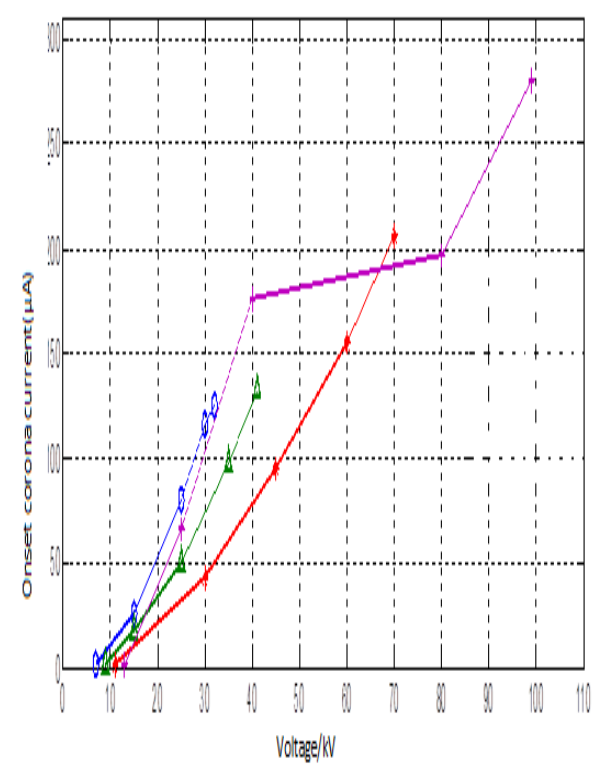

Figure 8: Pre - breakdown current as a function of potentials discharges in $\mathrm{SF}_{6}$ with distance $(0.4 \mathrm{~cm})$ and $(\mathrm{p}=0.5 \operatorname{bar}(\mathrm{O}), 1 \operatorname{bar}(\Delta)$, $2 \operatorname{bar}(*), 3 \operatorname{bar}(+))$

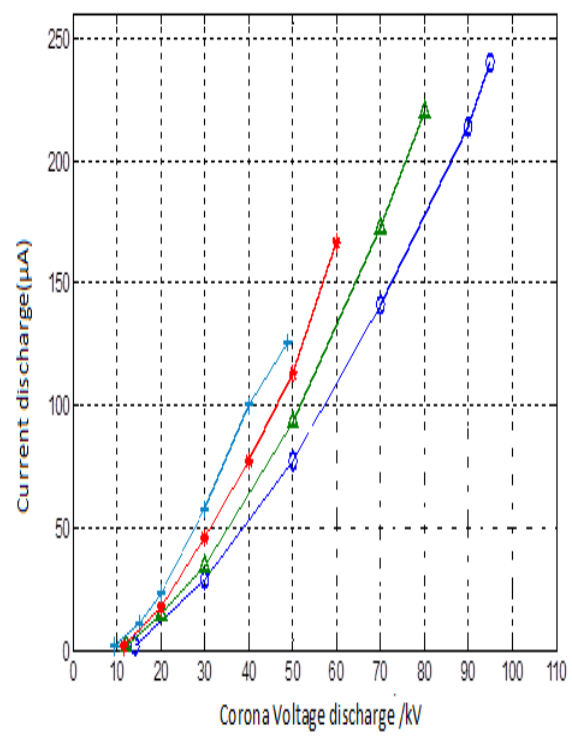

Figure 9: Pre - breakdown current as a function of potentials discharges in $\mathrm{SF}_{6}$ with distance $(0.5 \mathrm{~cm})$ and $(\mathrm{p}=1 \mathrm{bar}(+), 1.5 \mathrm{bar}(*)$, $2 \operatorname{bar}(\Delta), 2.5$ (O)bar)
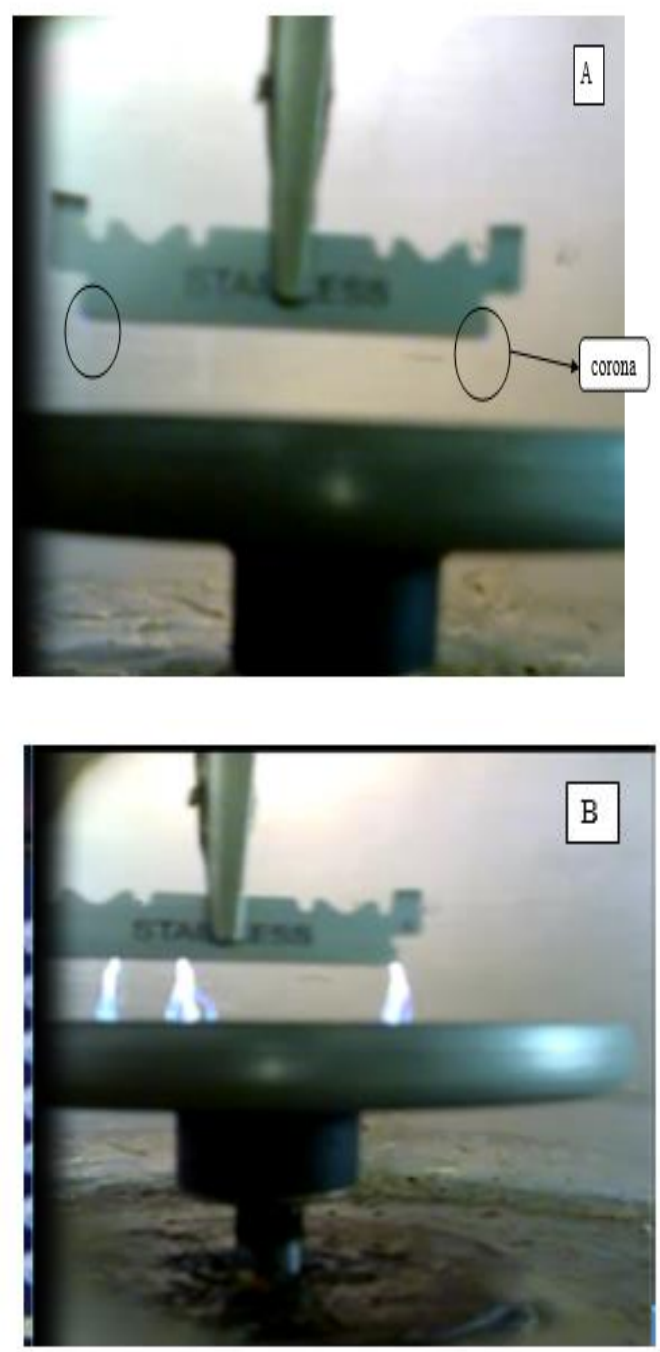

Figure 10 (A, B): Typical examples shows of corona inception and breakdown potentials 


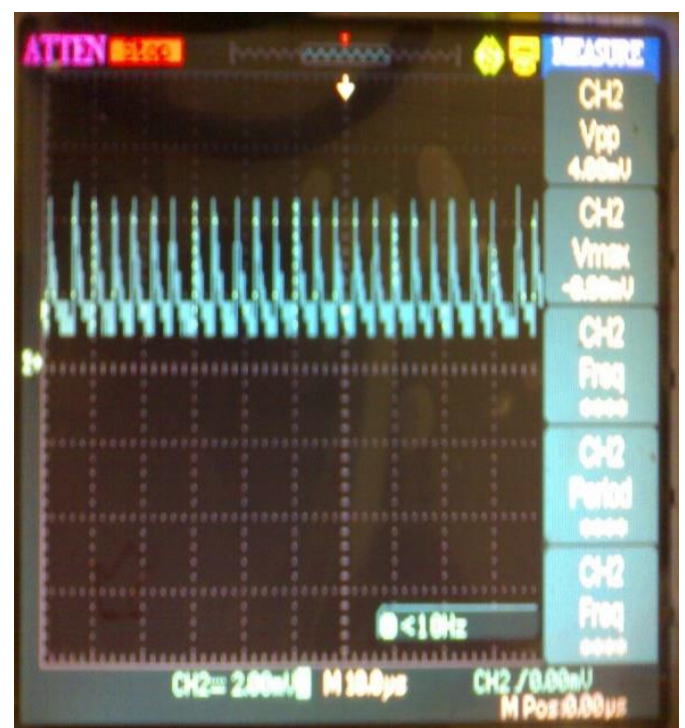

Figure 11: Typical record showing the time resolved pulses for sharp-to - plane geometry

\section{Conclusion}

1- The experimental study of a DC positive corona discharge phenomena and breakdown voltage fed by a Sulfur hexafluoride using high voltage generator over the range $110 \mathrm{kV}$.

That appear low corona inception voltage and breakdown while the current pre-breakdown or corona current is high, comparison with experimental data [11].

2- The addition of pure sulfur hexafluoride show significant influence on the corona voltage (CV) characteristics of a corona discharge even at high gas flow rates. This is caused by dissociative electron attachment to $\mathrm{SF}_{6}$ molecule. The dynamic process that is active through the whole discharge geometry and is dominant even at low pressure of gas . Also the sharp electrode area is probably most influential factor in the decreases of positive breakdown gradients

3- The actual experimental work deals with the investigation of a non-uniform sharp-to-plane electrode configuration under very high voltage apply, in order to describe the discharge development also typical voltage current measurements are used.

4- Corona pulses of currents exceeding $250 \mu \mathrm{A}$, corresponding to a applied voltage of $110 \mathrm{kV}$ for SF6 ,on other hand, under certain experimental conditions of gas pressure and electrode spacing results demonstrated a conspicuous deviation from Paschen's Law due to dominating streamer mechanism and electron attachment processes.

\section{References}

[1]. Loeb L. B., Electrical coronas, their basic physical mechanisms, Univ. Calif. Press, Berkeley, (1965).
[2]. Hassan jasim Mohammed Monte Carlo Simulation To Study Streamer Theory In Electronegative Gases, Diyala Journal of engineering Sciences, 02, December (2009), 3038.

[3]. E. Kuffel, W.S. Zaengl, J.Kuffel, High Voltage Engineering Fundamentals', E. Kuffel, W.S. Zaengl and J. Kuffel, (2000).

[4]. Hassan jasim Mohammed, Calculation Mobility \&Transverse Longitudinal Diffusion Coefficients In $\mathrm{SF}_{6}$ Gas Under The TOF Condition, Diala ,Jour, 39, (2009), 8494.

[5]. Gaseous Dielectrics, Proceedings of the International Symposium on Gaseous Dielectrics, Knoxville, Tennessee, USA, March 6-8 (1978).

[6]. A.S. Hasaani, Hassan jasim Mohammed "measurements Of corona discharge In non uniform Field Freon gap, Diyala Journal of Engineering Sciences, 07, (2014), 4761.

[7]. A.S. Hasaani ,R. R. Abdulla, Hassan jasim Mohammed, The Dynamic Processes In The Electrical Discharges Of $\mathrm{SF}_{6}$, Diyala Journal for Applied Researches (DJAR), 2, (2), (2006), 84101.

[8]. A.S.Hasaani, Corona And Breakdown Phenomena in SF6, Iraqi J. of science 8ti., 32, (2), (1991).

[9]. Hassan jasim Mohammed, Characterization Of The Dynamic Processes In the Electrical Discharge of sulfur Hexafluoride, M.SC. Thesis , University of Baghdad, 2001.

[10]. Abbass Mohd . Hassoun, The negative point to plane Breakdown in a free Electron gas, thesis, phd, university of California, Berkley, 1964.

[11]. Hassan jasim Mohammed, Corona Stabilization And Electric breakdown Of gases in nonuniform Fields, Phd. Thesis , University of Baghdad, 2013.

[12]. T. Nitta, thesis, contral research Laboratory Mitsubishi Electric corporation, Jappan, 1974.

[13]. O. Farish ,O. E. Ibraim, A. Kurimoto, Prebreakdown corona Processes in SF6 and SF6/N2 mixtures, Int. Symp. On High voltage engineering (1979). 